\title{
On the Development of Realistic Cross Layer Communication Protocol for Wireless Sensor Networks
}

\author{
Shalli Rani ${ }^{*}$, Jyoteesh Malhotra ${ }^{2}$, Rajneesh Talwar ${ }^{3}$ \\ ${ }^{1}$ SSDWIT College, Bathinda, India \\ ${ }^{2}$ Guru Nanak Dev University, Regional Campus, Jalandhar, India \\ ${ }^{3}$ CGC Engineering College, Chandigarh, India \\ Email: 'Shavi jindal@yahoo.com, jyoteesh@gmail.com, rtphdguidance@gmail.com
}

Received 20 April 2014; revised 15 May 2014; accepted 21 May 2014

Copyright (C) 2014 by authors and Scientific Research Publishing Inc.

This work is licensed under the Creative Commons Attribution International License (CC BY).

http://creativecommons.org/licenses/by/4.0/

(c) (i) Open Access

\begin{abstract}
Traditional protocols in wireless sensor networks follow the strict layering techniques which lead to the degradation in performance of the QoS (Quality of Service) parameters. Battery powered tiny sensor nodes with constraints of energy and guaranteed time require the efficient communication protocols with innovative and optimized approach to attain the objectives of WSN. Especially, these requirements become more stringent with the emergence of novel applications based on WSN. In order to find an optimized solution to this, cross layer-based approach which allows the cooperation, synchronization and communication among the layers, seems to be appropriate. In this paper, a realistic cross-layer protocol has been developed taking into considerations of MAC and Physical layer to further optimize the QoS parameters of already developed Energy Efficient Inter Cluster Coordination Protocol (EEICCP) at the Network layer level. In this paper we endeavor to concentrate on the enhancement of reliability parameter of $Q \circ S$ and results are validated through simulations done in MATLAB.
\end{abstract}

\section{Keywords}

Cross Layer, Routing Layer, MAC Layer, Physical Layer, Time, Frame Reception Probability

\section{Introduction}

Expansion of large scale sensor networks and investigation in different potential areas are the result of recent ad-

${ }^{*}$ Corresponding author 
vancements in wireless communication. Vast number of economical, tiny and limited battery-powered sensor nodes makes up wireless sensor networks, envisioned as sovereign and self organizing communication devices deployed throughout in dense physical space. WSN is constructive in congregation of information related to the humidity, environment, temperature etc., which is stirred to the base station (BS) for ancillary processing. Emergence of new and real time applications consigns pioneering challenges to WSN, and requires to be solved with novel, innovative approach and improved QoS (Quality of service) metrics such as time, reliability, scalability and energy efficiency. Intransience of network depends upon the limited energy of the nodes where network lifetime can be termed as the time till the first node depletes its energy. Layered architecture has been (OSI Model) successful in many areas but under above mentioned constraints a traditional network with no cooperation among different layers is inadequate. To exploit all potential benefits of the WSN, it is necessary to devise an optimized new technique to facilitate communication and coordination among the different layers of the network termed as Cross Layer Design (CLD). In previous work we investigate the impact of routing protocols with homogenous [1] and heterogeneous [2] approach and a new routing protocol for homogenous network [3] with hierarchy of clusters was developed. It proved to be successful in terms of energy efficiency, network lifetime and timely delivery. Only routing layer protocols [3]-[5] cannot attain all benefits of WSN. Routing layer performs the function of data transmission to the sink on the persistent path via single hop or multi hop. In long range wireless communication, multi hop technique not only drastically reduces the energy consumption [6] by short range communication of sensor nodes, but also reduces channel fading and signal propagation; so it is supreme idea to follow and also is used in EEICCP [3]. Jobs like end to end reliable data delivery (Transport layer), network throughput, delivery latency, data frame creation, error control (MAC layer), signal modulation and detection (Physical layer) etc. also need to be considered with routing layer functions to optimize the results in the area of WSN.

Organization of nodes in hierarchical form (cluster technique) and cross layer interaction between the layers can be a good and novel approach to optimize the QoS metrics. The aim of this research is to provide a platform for the enrichment of versatile WSN. To achieve this we tried the cross layer communication of routing layer of EEICCP with already developed MAC (Medium Access Control) and Physical layer [7] for 802.15.4 [8] and justified our inspiration of cross layer.

Reminder of the paper is as follows: In Section 2 related work with requirement of cross layer design is elaborated. In Section 3 EEICCP with cross layer design, introduction of previous work, and description of MAC and Physical layer communication and brief introduction of Markov Model are accomplished, followed by conclusion in Section 4.

\section{Related Work}

Development of diverse applications like habitat monitoring, global positioning system, environment monitoring etc. in the field of networking has escort the new research areas for enrichment of QoS metrics. QoS can be proficient by improving the hardware or the software layer by making it energy efficient, by new scalable options and proper utilization of resources in energy, time and reliability constrained environment. With the help of MAC and Physical (Phy) cross layer design (CLD), reliability in reception of safety messages has increased and PER (Probability Error Raito) decreased as mentioned in [9]. Routing layer consideration can make this protocol more effective as its aim is to transport vehicles under heavy load, then messages should be reached to the receiver in fast and reliable way. Without communication of network layer it seems difficult to be attained. Time, a delay parameter improvement has been shown by setting the priorities of the packets in the application and data link layer by CLD [10]. With sole parameter i.e. time not all the problems are solved in WSN, other parameter such as reliability may get influenced with fast delivery of data so other solutions could be find out. Priority of the data with delay parameter [11] has been optimized with sub layer of DLL (Data Link Layer) and Physical layer , and in our new strategy, model of 802.15.4 as in [11], is being considered but with the difference that routing protocol is EEICCP instead of AODV. Applications operating only on the routing protocols like [3]-[5], and [12]-[14] can't withstand in front of revolutionary challenges so cross layer (CL) approach development gave a new and innovative platform to the research scholars and scientists. New interfaces came into existence for the communication among the different layers to exploit the advantages of WSN [15] in which information can be shared among all the layers. In multi-hop environment it is necessary to reduce the end to end delay by having prior knowledge of the length of the route, interference among nodes etc. which affects the throughput of the protocol [16]. Performance of the system in term of error rate and throughput combined with adaptive modulation can be augmented [17] to enhance the performance of WSN. Provisioning of QoS metrics, 
function of routing, energy conservation are complementary goals, can be achieved by RSSI (Received Signal Strength Indication), interference control, back up nodes, shortest path etc. Responsibilities of all these factors depend upon different layers of the network model so it becomes necessary to breach the traditional system (OSI model) of layers to gain control over various shortcomings. Challenges faced by the new applications can be beaten by promising solution of CL (Cross Layer), there is need to develop the novel approach for feedback of each layer [18] and [19]. Interaction between seven abstract layers (OSI model) is scarce benevolent augment to the new thoughts to launch into the traditional paradigm, proving $\mathrm{CL}$ as the promising solution.

Our Contribution and motivation: A deep insight and study of Routing, MAC and Physical layers gave us the platform to build a protocol which could work as energy efficient with consistent performance. So we developed a novel protocol EEICCP for the routing layer which proved to be energy efficient on the concept, if short range communication is made possible as a substitute of long range between the sensor nodes then optimazation in the parameter of energy can be gained. This idea being adopted in designing of EEICCP and hence validation of protocol was proved in MATLAB. Only routing work could not fulfill all the objectives so revision of cross layer interaction among different layers motivated us to further improve the protocol by increasing its reliability by considering awakening and sleeping schedule of MAC and received signal strength of physical layer to make it viable for densely deployed network. So interaction of routing layer is made possible with MAC and Physical layer to calculate probability of frame reception, reliability etc. according to IEEE 802.15.4 model [8].

\section{EEICCP with Cross Layer Design}

In this section we epitomize the design of cross layer with EEICCP followed by Markov model adaptation results.

Introduction of Routing Protocol: Energy Efficient protocol with inter cluster [3] communication depends upon hierarchy of clusters for dissemination of data. Clusters communicate with each other with the help of cluster heads (CHs) and cluster coordinators (CCOs). In the set up phase, 1055 nodes are randomly deployed in the area of 200 by 200 sq. meter. Area according to x-axis and y-axis (at the difference of 20 meter on y axis one cluster is assumed) divided to form the cluster in the specific area and control all the communication in that area by one cluster head e.g. on the dimension of x-axis ( 0 - 200 meter) and y-axis $(0-20$ meter) first cluster was formed with first $\mathrm{CH}$ with no clusters underneath it. Then with difference of 20 meter (20 - 40, 40 - 60 etc.) on the dimension of $\mathrm{y}$-axis with $\mathrm{x}$-axis $(0-200$ meter for all clusters) other clusters boundaries were formed as shown in Figure 1 of [3]. Each Cluster has one $\mathrm{CH}$ for gathering of data from all nodes of corresponding cluster and CCOs in each cluster vary according to the incremental approach $\left(\mathrm{k}^{*}(\mathrm{k}+1) / 2\right)$ where $\mathrm{k}$ is the number of clusters. So in the area measurement of vertically 0 - 20 meter there is no CCO because it is assumed to be at the highest depth as like tree data structure where leaf nodes have no child nodes. Selection sort algorithm is used to sort the nodes according to their distance from base station (node with highest distance assumed to be at highest depth). But on its top one CCO is established to transfer its $\mathrm{CH}$ data to the next CCO of next cluster until it reaches to the BS (Base Station). We have assumed ten clusters, $10 \mathrm{CHs}$ and $45 \mathrm{CCOs}$ with election and data transfer phase for 1055 nodes. In the election phase, $\mathrm{CHs}$ and CCOs are selected randomly according to the proposed strategy and Ids of all CHs and CCOs are exchanged to announce their election with broadcasting of messages to the nodes for identification of their respective clusters. Number of CHs and CCOs depends upon the number of clusters so they are predefined and static. First order radio model employed in LEACH [4] and HCR [5] is used to calculate the energy consumption for the long and short distance in reception and transmission of data. When number of nodes is increased than 1055 then area is divided into subareas and transfer algorithm of EEICCP is repeated in both subareas. EEICCP showed improvement in two parameters i.e. energy [3] and time [22] over LEACH and HCR as shown in Table 1.

\subsection{MAC and Physical Layer Communication Model}

Key responsibilities of MAC layer are collision avoidance, adaptability, scalability, channel utilization, latency,

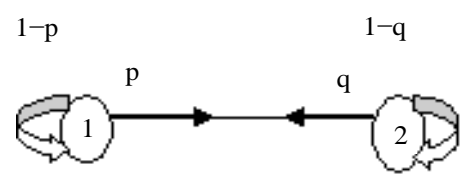

Figure 1. Two state Markov chain. 
Table 1. Improvement of EEICCP in QoS metrics.

\begin{tabular}{ccc}
\hline Parameter & EEICCP $<$ HCR & EEICCP $<$ LEACH \\
\hline Energy & $43 \%$ & $50 \%$ \\
Time & $70 \%$ & $43 \%$ \\
\hline
\end{tabular}

efficiently sharing of resources [6]. Two most common fundamental MAC protocols used for sensor networks are time division multiple access (TDMA) and collision sense multiple access (CSMA). Major benefit of TDMA is collision avoidance but it introduces more delay under light traffic load and it increases complexity in terms of time synchronization between sensor nodes making it difficult to accommodate changes in network. In contrast CSMA increases delay under higher traffic load [6] but suitable under lower traffic load because of contention for the channel. But either of both could be used with other protocols for the betterment of results for different requirements of different applications. Main duty of Physical layer is to generate signals from bit streams received from DLL. Major issues to be handled by this layer are signal modulation, data encryption, signal detection etc. When both Physical and MAC layer interacts with each other performance of the model for WSN is increased. In recent times MAC and PHY layer cross layer design revolves through novel techniques such as optimization in signal modulation, frame reception probability, reliability, cross layer scheduling etc. Power control (physical layer) and back off (MAC) algorithms in cross layer protocol [20] performed well and optimized parameters of delay, fairness and throughput. Design of cross layer can be implemented in two ways that are tightly coupled and loosely coupled [21]. In later technique optimization is done on one layer but by considering the aspects of other layers i.e. information about parameters is exchanged between the layers. For example information about collision from MAC layer and frame reception probability from PHY layer can be passed to the routing layer. Routing layer can pass the distance of the nodes to the MAC and PHY layer for calculation of both parameters. In contrast, tightly coupled systems not only exchange information among layers but new routing algorithm is developed for further optimization; on the basis of parameters of other layers. As by considering the interference of the nodes, routing path can be changed among the nodes [21]. MAC and Physical layer are close to each other so cross layer design is common on both layers. [7] introduced a MATLAB model on the basis of 802.15.4 model of IEEE. This model basically shows the communication between MAC layer and Physical layer which includes path loss and shadowing effects. It includes a function ZunPhyModel which operates on physical layer for the calculation of probability of frame reception. This value is returned to the MAC layer with the help of rs2m_802154_mac function to find out the reliability against signal to noise ratio (SNR) and radio parameters. In this model dblquad, an inbuilt function of MATLAB is used over distance and shadowing standard to know the probability of successful frame reception which is the consequence of SNR, path loss, packet error and packet coding rate. As IEEE 802.15.4 model rely on the Markov chain Model [22] to measure the backoff counter, retransmission counter and $\mathrm{M} / \mathrm{M} / 1 / \mathrm{K}$ queue model that envisage the consequence of a restricted buffer in the station [7]. With the help of generalized Markov model [23], reliability, delay, average back off periods are calculated in [7]. Some inputs of CC1000 [24] are used in ZunPhyModel. This model has demonstrated the frame reception versus distance to measure the reliability per node.

To facilitate the cross layer benefits of [7] we tried to use the routing layer of EEICCP on its top. As in loosely coupled cross layer design only information among the layers is exchanged so same is adopted in our present work.

\subsection{Markov Chain Model}

A discrete time chain which does not depend upon the past but only on the present is known as Markov chain. Binachi model [24] described Markov chain model for the MAC layer. It can be two state (Figure 1) or multistate chain. The transition matrix for two state models can be described as Equation (1). In the same way n state Markov chain is evaluated for the selection of new values randomly. A three state Markov chain has been used in [23] for backoff counter, backoff stage and retransmission counter for the transmission of a packet. Saturated traffic is assumed in [7], same is done in EEICCP. EEICCP assumes that a node always has data to send. Non null one step transitions probabilities are described in the Markov chain of [25]. Cases of beginning of slot time, new packet transmission after the successful transmission of first packet etc. are discussed. Study of this model was distinguished and generalized in the [23] and later it inspired [7] to carry out this work on the PHY layer. 
Equations (16), (17) and (18) of [23] were reconsidered in [7] which worked on the probability that a node had always data to send.

$$
r=\left(\begin{array}{cc}
1-p & p \\
q & 1-q
\end{array}\right)
$$

Model of [23] was used in [7] with one exception of saturated traffic. As EEICCP also considers traffic to be saturated so this model is suitable for our protocol.

\subsection{Cross Layer Design of EEICCP}

In the earlier work of EEICCP, new routing algorithm was developed and proved to be energy efficient. After energy efficient algorithm development another parameter i.e. time [22] was calculated and again EEICCP was found optimized in terms of delay parameter. To exploit the benefits of CLD, we investigate the protocols based on cross layer designs and found that loosely coupled design of cross layer will best fit with already developed routing protocol. As in this type of design, only information is exchanged among the layers about specific parameters of different layers. We used the routing layer on the top of MAC and Physical layer model of [7] and Information about the distance of nodes from their respective $\mathrm{CH}$ of first cluster in EEICCP is passed to ZunPhyModel function of [7]. Minimum and maximum distance of the nodes is taken 1 meter and 20 meter in [7]. For the communication of our routing protocol (EEICCP) with joint model of IEEE 802.15.4 [7], the distance parameter of the nodes (in range of 1 meter to 20 meter) of first cluster is passed to the Zun Phy Model [7] function. Number of stations in [18] are 10 but nodes are deployed randomly in EEICCP and clusters are made according to dimension of $\mathrm{x}$-axis and y-axis that is why nodes (Number of stations) are not fixed in any cluster. We developed a function to count the nodes in the specific range which is assumed in [7] (1 meter - 20 meter) in a cluster and passed the values of their distance to function defined in [7] (ZunPhyModel defined at Physical layer) calculate the frame reception probability which is passed to another function [7] (Mac Layer Function) to calculate the total execution time, wait time, reliability, failure probability, throughput, channel access failure probability as shown in Appendix from Figures 2-8. Total execution time for 17 nodes of EEICCP is 21.2 seconds. In Figure 2 alpha and beta probabilities are shown to find out that a channel is busy or not during first and second sensing. In Figure 3 channel access failure probability (Pcf) and packet discarded due to retry limits probability (Pcr) shows that nodes of EEICCP do not fail due to retry limits but channel access failure increases as more number of nodes retry to transmit the data. Instantaneous throughput, average throughput and reliability decreases as load per node is increased (Figures 5-7). Failure probability and average waiting time increased with increase in offered load (Figure 4 and Figure 8). All these figures are on the basis of already developed cross layer model [7] by passing the distance parameter and number of nodes of EEICCP to the physical and MAC layer of [7].

\section{Conclusion}

In this paper we have examined the cross layer communication of our routing layer with already developed MAC and Physical layer MATLAB model. In the previous work we compared results of energy and time with LEACH and HCR and found EEICCP optimized over both. Other most necessary parameters i.e. reliability, average waiting time, and throughput have been calculated by adopting cross layer communication with routing layer of EEICCP. EEICCP is better than HCR and LEACH as the parameters which were not considered in LEACH and HCR, are calculated in EEICCP. Our protocol considered not only newly developed routing strategy but also MAC and Physical layer parameters to know other factors to calculate the reliability of the nodes. In all of the simulations the EEICCP outperforms the LEACH and HCR and furthermore it is not confined only to the routing work as like LEACH and HCR but also includes cross layer communication with MAC and Physical layer. Till now, with inter-cluster communication EEICCP has improved parameter of energy and time and also achieved the reliability parameter by cross layer design, in future we will also consider intra-cluster communication in efficient way for further improvement of QoS metrics in WSN.

\section{References}

[1] Chen, G., Li, C., Ye, M. and Wu, J. (2009) An Unequal Cluster-Based Routing Protocol in Wireless Sensor Networks. 
Wireless Networks, 15, 193-207. http://dx.doi.org/10.1007/s11276-007-0035-8

[2] Matta, S.I. and Bestavros, A. (2004) SEP: A Stable Election Protocol for Clustered Heterogeneous Wireless Sensor Networks. Technical Report BUCS-TR-2004-022G.

http://open.bu.edu/xmlui/bitstream/handle/2144/1548/2004-022-sep.pdf?sequence=1

[3] Rani, S., Malhotra, J. and Talwar, R. (2013) EEICCP-Energy Efficient Protocol for Wireless Sensor Networks. Wireless Sensor Networks, 5, 127-136. http://dx.doi.org/10.4236/wsn.2013.57016

[4] Heinzelman, W., Chandrakasan, A. and Balakrishnan, H. (2000) Energy-Efficient Communication Protocol for Wireless Microsensor Networks. Proceedings of the 33rd Hawaii International Conference on System Sciences, Maui, 4-7 January 2000. http://dx.doi.org/10.1109/HICSS.2000.926982

[5] Hussain, S. and Matin, A.W. (2006) Hierarchical Cluster-Based Routing in Wireless Sensor Networks. Proceedings of International Conference of Information Processing in Sensor Networks (IPSN), Nashville, 19-21 April 2006.

[6] Zheng, J. and Jamalipour, A. (2009) Wireless Sensor Networks-A Network Perspective. John Wiley \& Sons, Hoboken.

[7] Zayani, M.-H., Gauthier, V. and Zeghlache, D. (2011) A Joint Model for IEEE 802.15.4 Physical and Medium Access Control Layers. 7th International Conference on Wireless Communications and Mobile Computing Conference (IWCMC), Istanbul, 4-8 July 2011, 814-819. http://dx.doi.org/10.1109/IWCMC.2011.5982651

[8] IEEE (2006) IEEE Std 802.15.4-2996, September, Part 15.4: Wireless Medium Access Control (MAC) and Physical Layer (PHY) Specifications for Low-Rate Wireless Personal Area Networks (WPANs). http://standards.ieee.org/getieee802/download/802.15.4-2006.pdf

[9] Cassidy, W.G., Jaber, N. and Tepe, K.E. (2012) Cross Layer MAC and PHY Architecture for Collision Correction of DSRC Safety Messages. IEEE 5th International Conference on New Technologies, Mobility and Security (NTMS), Istanbul, 7-10 May 2012, 1-5.

[10] Mohaghegh, M., Manford, C. and Sarrafzadeh, A. (2011) Cross-Layer Optimization for Quality of Service Support in Wireless Sensor Networks. IEEE 3rd International Conference on Communication Software and Networks (ICCSN), Xi’an, 27-29 May 2011, 528-533.

[11] Al-Anbagi, I.S., Erol-Kantarci, M. and Mouftah, H.T. (2012) Fairness in Delay-Aware Cross Layer Data Transmission Scheme for Wireless Sensor Networks. 26th Biennial Symposium on Communications (QBSC), Kingston, 28-29 May 2012, 146-149.

[12] Ye, M., Li, C., Chen, G. and Wu, J. (2005) EECS: An Energy Efficient Clustering Scheme in Wireless Sensor Networks. 24th IEEE International Performance, Computing, and Communications Conference, Phoenix, 7-9 April 2005, 535-540.

[13] Xin, G., Yang, W.H. and De Gang, B. (2008) EECHA-An Energy Efficient Hierarchical Clustering Algorithm for Wireless Sensor Networks. Information Technology Journal, 7, 245-252. http://dx.doi.org/10.3923/itj.2008.245.252

[14] Younis, O. and Fahmy, S. (2004) HEED: A Hybrid, Energy-Efficient, Distributed Clustering Approach for Ad-Hoc Sensor Networks. IEEE Transaction on Mobile Computing, 3, 366-379. http://dx.doi.org/10.1109/TMC.2004.41

[15] Hefeida, M., Shen, M., Kshemkalyani, A. and Khokhar, A. (2012) Cross-Layer Protocols for WSNs: A Simple Design and Simulation Paradigm. 8th International Wireless Communications and Mobile Computing Conference (IWCMC 2012), Limassol, 27-31 August 2012, 844-849.

[16] Cheng, M., Ye, Q.M. and Cai, L. (2013) Cross-Layer Schemes for Reducing Delay in Multihop Wireless Networks. IEEE Transactions on Communications, 12, 928-937.

[17] Go, K.C., Cha, J.R., Oh, S.K. and Kim, J.-H. (2013) End-to-End Performance Analysis Based on Cross-Layer Retransmission Scheme in Wireless Communication System. 2013 International Conference on Information Networking (ICOIN), Bangkok, 28-30 January 2013, 141-144.

[18] Fu, B., Xiao, Y., Deng, H. and Zeng, H. (2013) A Survey of Cross-Layer Designs in Wireless Networks. IEEE Communications Surveys \& Tutorials, 16, 110-126.

[19] Mendes, L.D.P. and Rodrigues, J.J.P.C. (2011) A Survey on Cross-Layer Solutions for Wireless Sensor Networks. Elsevier Journal of Network and Computer Applications, 34, 523-534.

[20] Sylwia, R. and Chris B. (2009) Cross Layer PHY-MAC Protocol for Wireless Static and Mobile Ad Hoc Networks. EURASIP Journal on Advances in Signal Processing-Special Issue on Signal Processing Applications in Network Intrusion Detection Systems, 2009, Article ID: 278041.

[21] Akyildiz, I.F. and Wang, X.D. (2008) Cross-Layer Design in Wireless Mesh Networks. IEEE Transactions on Vehicular Technology, 57, 1061-1076.

[22] Shalli, R., Jyoteesh, M. and Rajneesh, T. (2014) Energy Efficient Protocol for Densely Deployed Homogeneous Network. International Conference on Issues and Challenges in Intelligent Computing Techniques, Ghaziabad, 7-8 Febru- 
ary 2014, 292-298. http://dx.doi.org/10.1109/ICICICT.2014.6781295

[23] Park, P., Di Marco, P., Soldati, P., Fischione, C. and Johansson, K.H. (2009) A Generalized Markov Chain Model for Effective Analysis of Slotted IEEE 802.15.4. Mobile Adhoc and Sensor Systems. IEEE 6th International Conference on Mobile Adhoc and Sensor Systems, Macau, 12-15 October 2009, 130-139.

[24] Texas Instruments (2009) CC1000 Single Chip Very Low Power RF Transceiver. http://focus.ti.com/lit/ds/symlink/cc1000.pdf

[25] Bianchi, G. (2000) Performance Analysis of the IEEE 802.11 Distributed Coordination Function. IEEE Journal on Selected Areas in Communications, 18, 535-547. http://dx.doi.org/10.1109/49.840210 


\section{Appendix}

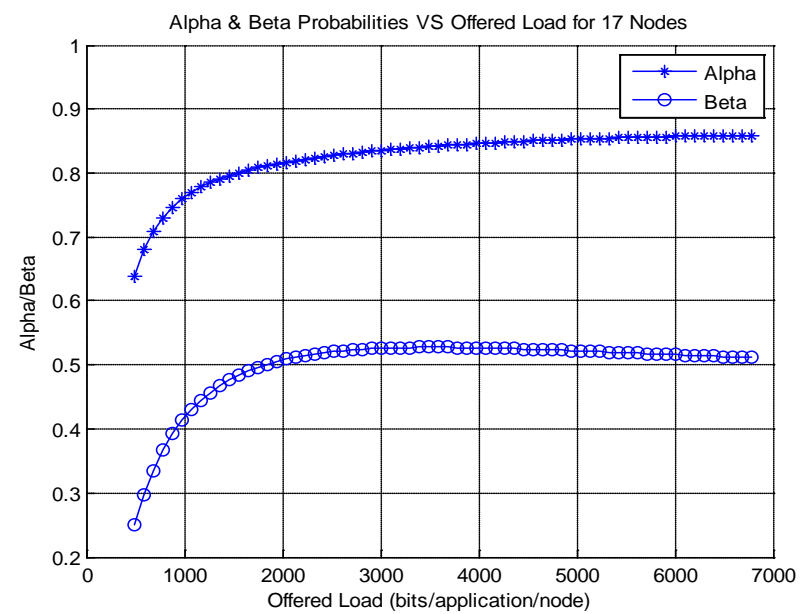

Figure 2. For channel busy or free during first and second sensing (Alpha and Beta).

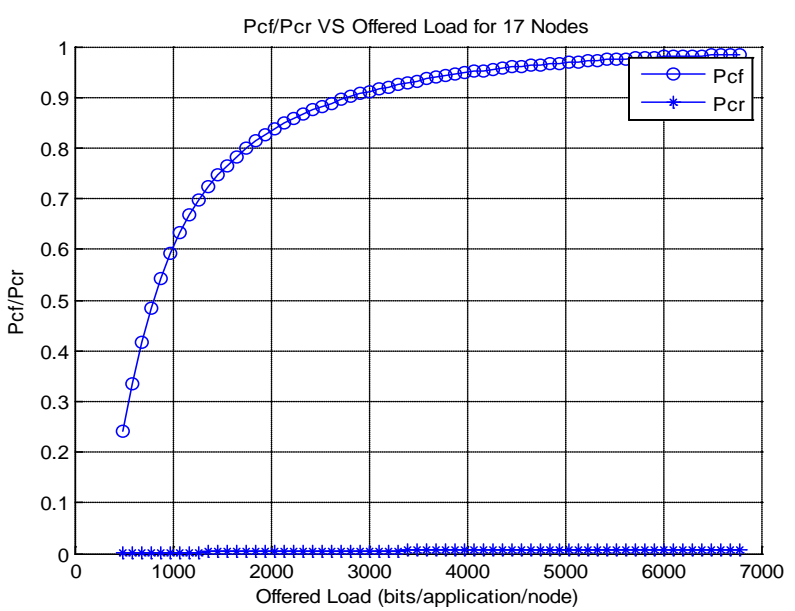

Figure 3. Channel access failure and packet discarded rate (Pcf and Pcr).

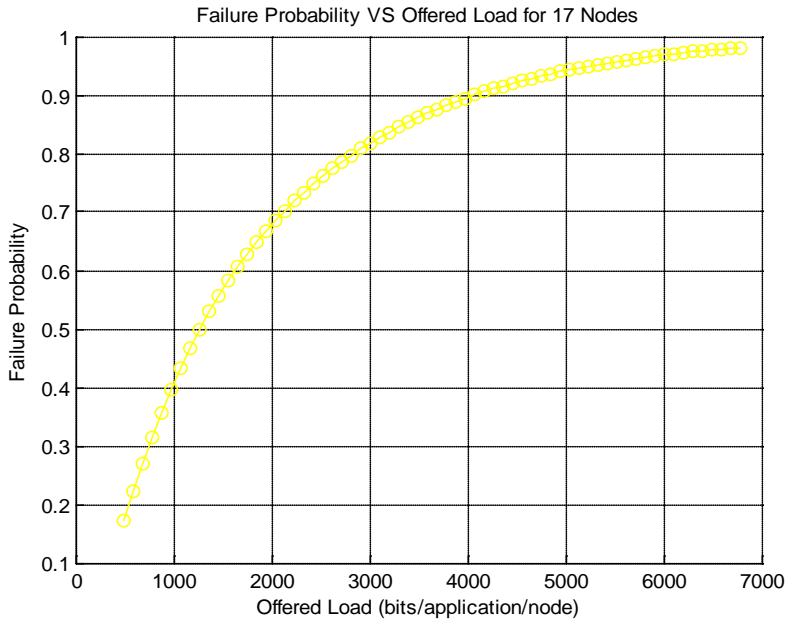

Figure 4. Failure probability of node. 


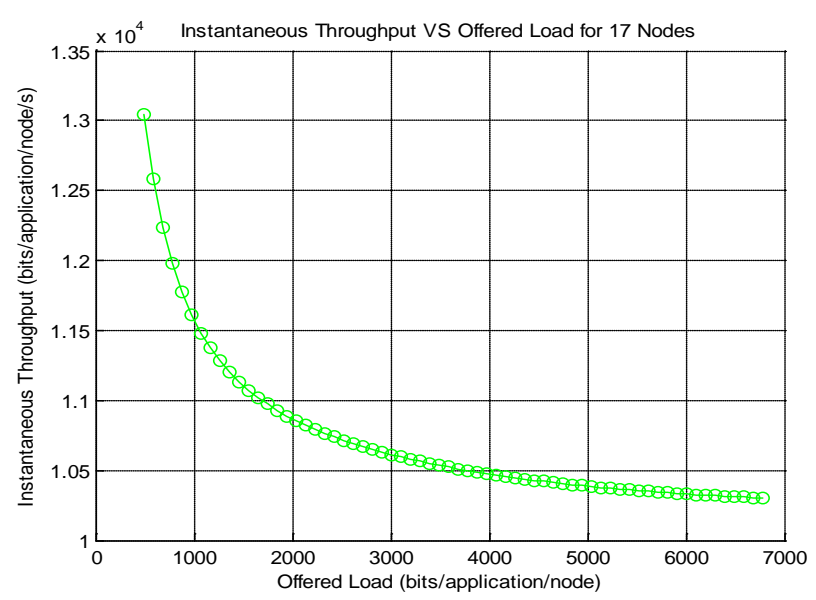

Figure 5. Instantaneous throughput for increased load.

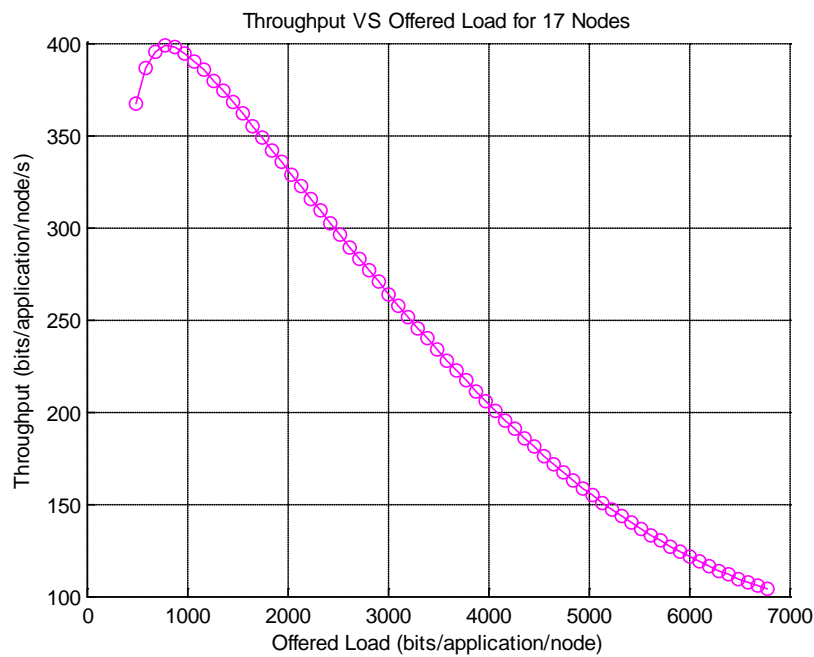

Figure 6. Average throughput.

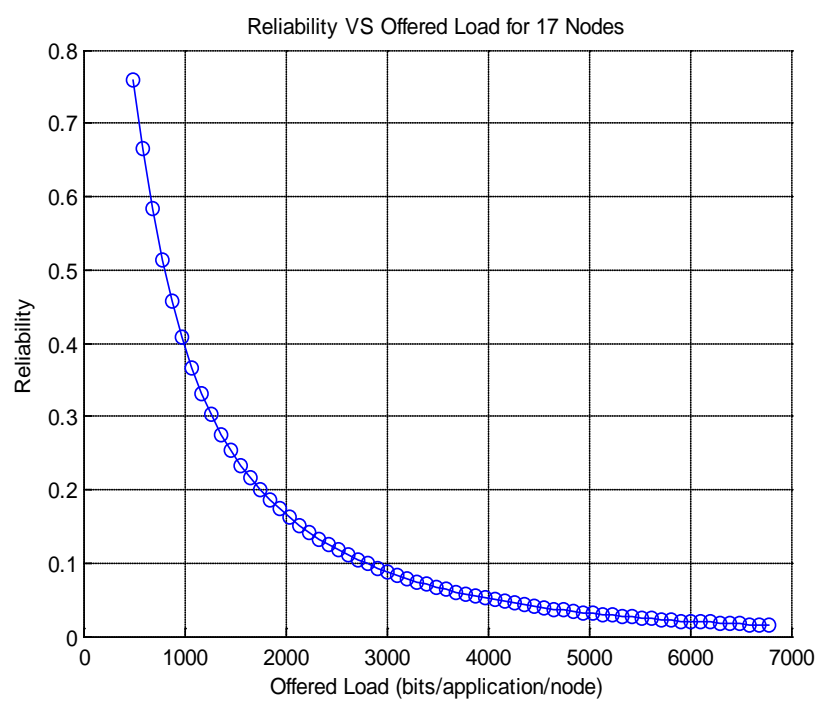

Figure 7. Reliability against increased load. 


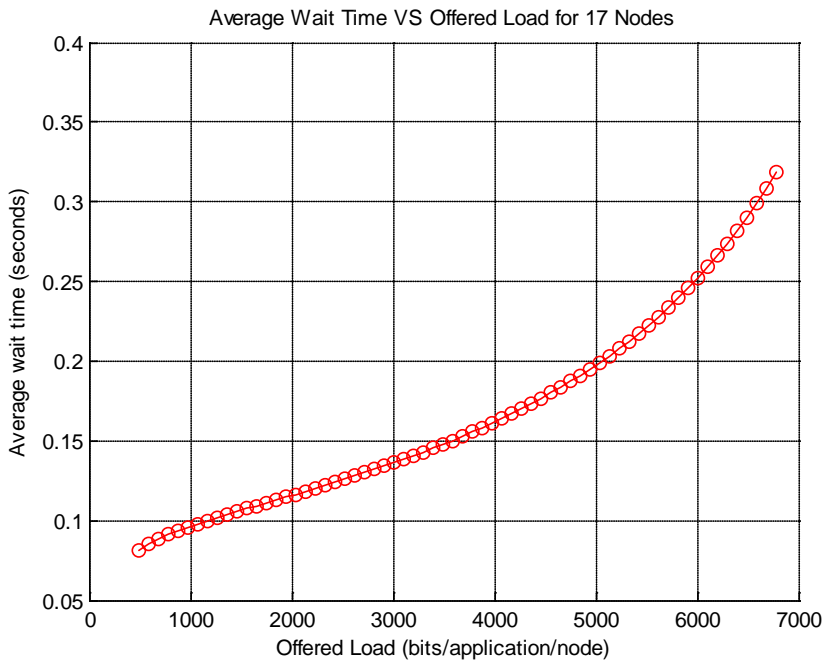

Figure 8. Average wait time against increased load. 